Palabras clave: Adolescencia, Familia, Olson, Edad.

\title{
¿Juega la familia un papel clave en la salud durante la adolescencia?
}

\author{
Nicolas Zdanowicz, Psychiatric D. \\ Janne Pascal, Prof Psychol \\ Christine Reynaert, Prof Med \\ Université Catholique de Louvain, \\ Clinique de Mont-Godinne, Service de \\ psychosomatique, 5530 Yvoir \\ BELGIUM
}

\begin{abstract}
RESUMEN - Objetivos: Examinar las diferencias en los lazos familiares entre un grupo "normal" de sujetos jóvenes de edades entre los 13 y los 25 años y un grupo con trastornos mentales.

Método: 814 sujetos jóvenes "normales" completaron el cuestionario Olson sobre su propia familia. Éste grupo se comparó con otro de 358 jóvenes que sufrían trastornos mentales.

Resultados: Los jóvenes del grupo sano venían de familias que eran notablemente más cohesionadas y adaptables que las del grupo de enfermos. También se documentó que los jóvenes del grupo de pacientes provenían de categorías de familias "desacopladas-estructuradas" y "desacopladas-rígidas", con los criterios definidos por Olson. Mientras que el género no tuvo influencia en estos dos grupos, la edad determinó una progresiva reducción en la cohesión en el grupo de sujetos jóvenes normales.

Conclusiones: Mientras la adolescencia “normal” está marcada por una disminución en la cohesión familiar con la edad, los adolescentes con trastorno mental provenían de familias que eran menos cohesionadas y adaptables desde el principio, comparados con los jóvenes "normales".
\end{abstract}

\section{Introducción}

El modelo circunflejo diseñado por Olson \& Killorin (1985) trata de evaluar dos dimensiones (dos ejes) de funcionamiento en un sistema de relación: cohesión y adaptabilidad. La cohesión se define por "lazos emocionales que cada miembro de la familia desarrolla con otros miembros de la familia". La adaptabilidad es la "habilidad del sistema marital o familiar para cambiar su estructura de liderazgo, y su papel en las relaciones y las reglas de esas relaciones, en respuesta a situaciones estresantes o cambios". Aunque el Cuestionario de Olson puede ser aplicado de dos maneras (como una valoración independiente llevada a cabo 
por especialistas o como auto-informe), ambas se refieren al mismo modelo biaxial. En este estudio hemos utilizado el modelo de auto-informe. Esta versión, conocida como FACES III (Family Adaptability and Cohesion Evaluation Scale, Escala de Evaluación de la Adaptabilidad y Cohesión Familiar, Olson, 1986), permite una valoración rápida y empírica de los dos ejes, describiendo ambos un estilo de interacción y estilo en el centro del sistema. El cuestionario comprende veinte preguntas (diez por eje) que el sujeto debe responder en términos de frecuencia (de 1 a 5). Cuando se suman las puntuaciones se obtienen dos puntuaciones globales (mínimo 10, máximo 50), una para adaptabilidad y otra para cohesión. El modelo ha sido diseñado de tal manera que la "salud" familiar se puede determinar mediante esas dos dimensiones desde los valores medios de los dos ejes, hablando de "separado-conectado" para cohesión y de "rígido-flexible" para adaptabilidad. Así, en este modelo la "salud" descansa en el punto medio, siendo una normalidad equilibrada. La ventaja de este sistema de clasificación es que evita una clasificación lineal estricta como la que se utilizó, por ejemplo, en el modelo de Beavers-Timberlawn (Beavers, 1982) en el que en una sola línea se ubicaban desde el estado patológico hasta el sano. Aunque el sistema biaxial de Olson tiene ciertamente beneficios adicionales, sin embargo ha sido criticado por ser demasiado normativo, bi-normativo en lugar de mononormativo (Reynaert et al. 1991, 1993). La representación grafica del modelo se compone de dos ejes perpendiculares con adaptabilidad en el eje "Y" y cohesión en el eje "X". Cada dimensión esta dividida en cuatro grados, resultando dieciséis subtipos diferentes, que pueden ser utilizados para caracterizar la familia. Además, tres zonas esbozan las siguientes características de funcionamiento: equilibrado, medio y extremo.
Es imposible revisar la bibliografía de todas las áreas en las que ha sido aplicado el FACES III, por ser demasiado extensa. Éstas van desde el estudio de la respuesta familiar ante un niño que pasa un tiempo en cuidados intensivos (Youngbloud et al. 1995) hasta la probabilidad de recaída en la esquizofrenia debida a las interacciones familiares (King et al. 1995). En nuestro tema específico, los vínculos entre adolescente-familia y enfermedad, existe un considerable número de estudios que investigan las relaciones entre una patología dada y el funcionamiento familiar (Tabla I).

Nuestro objetivo en este estudio es, más o menos, opuesto al de los listados en la Tabla I. En lugar de comenzar con una población con una patología dada y compararla con una población "sana", partimos de una población de adolescentes "sanos" y la comparamos con una muestra de enfermos. De hecho, la pregunta es si las características de la familia representan una susceptibilidad para una patología dada o, como sugieren los resultado de Prenge et al. (1992), las características de la familia representan una mayor sensibilidad hacia la enfermedad. Finalmente, nos propusimos investigar la influencia del género y de la edad.

\section{Método}

Este estudio se llevó a cabo entre diciembre de 1998 y junio de 1999. Además del FACES III, los sujetos también completaron el Locus de Control de Salud Multidimensional (Multidimensional Health Locus of Control-MHLC, Wallston et al. 1978), que examina las creencias de una persona con respecto a la responsabilidad que tiene en determinar su propio estado de salud. Esta encuesta suplementaria fue incluída para 
Tabla I

FACES III y adolescencia: Revisión bibliográfica

\begin{tabular}{|c|c|c|c|}
\hline Autores & Tema de estudio & Edad & Correlaciones con el modelo de Olson \\
\hline Coburn and al (1989) & Bulimia & $\begin{array}{l}\text { Mujeres estudiantes } \\
\text { universitarias }\end{array}$ & $\begin{array}{l}\text { Las pacientes bulímicas tienen una } \\
\text { pobre cohesión familiar. } \\
\text { La adaptabilidad no juega ningún } \\
\text { papel. }\end{array}$ \\
\hline Kashani and al (1995) & Depresión & $<13$ años & $\begin{array}{l}\text { Lo niños deprimidos tienen familias } \\
\text { desligadas. } \\
\text { La adaptabilidad no juega ningún } \\
\text { papel. }\end{array}$ \\
\hline Lawler and al (1990) & Control diabético & 15-18 años & $\begin{array}{l}\text { Hay una correlación entre control } \\
\text { diabético y cohesión; los pacientes } \\
\text { desligados obtienen los resultados } \\
\text { más pobres. }\end{array}$ \\
\hline Lundholm and al (1991) & $\begin{array}{l}\text { Trastornos de la } \\
\text { conducta alimentaria }\end{array}$ & $\begin{array}{l}\text { Mujeres estudiantes } \\
\text { universitarias }\end{array}$ & $\begin{array}{l}\text { Las estudiantes con trastornos } \\
\text { de conducta alimentaria } \\
\text { tienen familias extremas. }\end{array}$ \\
\hline Michaels and al (1990) & $\begin{array}{l}\text { Trastornos } \\
\text { de aprendizaje }\end{array}$ & 6-12 años & $\begin{array}{l}\text { Los niños con trastornos } \\
\text { de aprendizaje } \\
\text { tienen familias extremas. }\end{array}$ \\
\hline Prange and al (1992) & $\begin{array}{l}\text { Trastornos } \\
\text { afectivos }\end{array}$ & 12-18 años & $\begin{array}{l}\text { Los adolescentes con trastornos } \\
\text { afectivos tienen familias desligadas. } \\
\text { Una cohesión débil correlaciona con } \\
\text { más psicopatología. }\end{array}$ \\
\hline Tubiana and al (1998) & Control diabético & 7-13 años & $\begin{array}{l}\text { Cohesión y adaptabilidad están pro- } \\
\text { porcionalmente relacionadas con } \\
\text { cumplimentación del tratamiento. } \\
\text { Familias rígidas y desligadas = pobre } \\
\text { control diabético. }\end{array}$ \\
\hline
\end{tabular}

permitir correlacionar los lazos familiares con la MHLC en futuros estudios, si se confirman nuestras hipótesis (Zdanowicz et al. 2002). Utilizamos la versión francesa de los cuestionarios FACES III de Olson y el MHLC, traducidos por Fontaine (1985) y Janne \& Mortreu (1989), respectivamente.

\section{Sujetos participantes y procedimiento}

El grupo "sano" fue reclutado de diferentes maneras para asegurar diferentes grupos de edad. El primer reclutamiento se llevó a cabo en estudiantes de los seis años de educación secundaria obligatoria (en otras palabras, desde los 12 al los 18 años de edad) en tres diferentes institutos de secundaria de la provincia de Namur (Bélgica). Pedimos a los jóvenes, en presencia de un psiquiatra evaluador, que facilitaran información sociodemográfica (edad, nombre, sexo, nivel de escolarización y nacionalidad), y que completaran la escala de Olson para su propia familia y para su familia ideal, y el MLHC. El segundo reclutamiento se llevó a cabo en la Universidad Católica de Lovaina (UCL), en la Facultad de Psicología. Por defecto, nuestro grupo "sano" se considera "normal". Sin embargo, como este grupo no fue reclutado en un centro de salud o en un hospital, no podemos descartar que algunos de estos jóvenes pudieran estar en tratamiento ambulatorio o haber sido hospitalizados por alguna razón. 
El grupo de adolescentes "enfermos" se configuró utilizando datos informatizados sistemáticamente desde 1990 de pacientes de los departamentos de medicina psicosomática y psicopatología de la Clínica MontGodine de la UCL. La inclusión de sujetos se cerró en diciembre de 1998. Al comienzo de la hospitalización, y bajo la supervisión de un psicólogo, los pacientes completaron los datos generales, que incluían edad y fecha de nacimiento, un cuestionario MHLC y un cuestionario de Olson. Para todos los pacientes era su primera hospitalización voluntaria y no venían de ningún tipo de dispositivo residencial. En un intento de controlar el efecto de la duración del período de reclutamiento, los resultados se controlaron tomando la fecha de admisión como una covariable. Optamos por tener en cuenta el diagnóstico para mantener la naturaleza trans-nosográfica de nuestras hipótesis. De cualquier modo, en la Tabla II listamos los diagnósticos con propósitos informativos.

Independientemente de si pertenecían al grupo sano o al "enfermo", los candidatos tenían que cumplir los siguientes criterios:

Tabla II

Diagnósticos del eje I del DSM-IV (1994)

\begin{tabular}{lrc} 
Eje I & N & $\%$ \\
\hline Número de diagnósticos para 358 adolescentes & 464 & 100 \\
Sin diagnóstico & 30 & 8,20 \\
Más de un diagnóstico & 121 & 33,8 \\
Trastorno depresivo & 179 & 37 \\
Trastornos de ansiedad & 71 & 14,7 \\
Trastornos relacionado con alucinógenos & 64 & 13,2 \\
Trastorno por déficit de atención y conductas desadaptativas & 49 & 10,1 \\
Esquizofrenia y otros trastornos psicóticos & 27 & 5,6 \\
Trastornos de la conducta alimentaria & 24 & 4,9 \\
Trastornos relacionados con el alcohol & 23 & 4,8 \\
Trastornos somatomorfos & 18 & 3,7 \\
Trastornos de adaptación & 9 & 1,8 \\
Trastornos de aprendizaje & 8 & 1,6 \\
Trastornos por abuso de varias sustancias & 7 & 1,4 \\
Trastornos ficticios & 1 & 0,2 \\
Retraso mental & 1 & 0,2 \\
\hline
\end{tabular}

1. Edad entre los 13 y los 25 años.

2. Estado marital: soltero o en pareja sin cohabitar.

3. Estado socio-profesional: desempleado, sin profesión y/o estudiante.

Estos tres criterios son los propuestos por la Organización Mundial de la Salud (1994) para describir la "situación de la adolescencia”. Para garantizar la homogeneidad de nuestra población, los sujetos debían ser estudiantes, caucásicos y francófonos.

\section{Análisis y presentación de resultados}

El análisis estadístico se efectuó mediante el programa informático SPSS para Windows 95/98/NT Modelos Avanzados 9.0S. Debido al significativo número de observa- 
ciones y a la necesidad de analizar la influencia de ciertas covariables, utilizamos pruebas paramétricas. Se utilizó la prueba chi-cuadrado de Pearson comparar los porcentajes. Las correlaciones entre las variables constantes se determinaron mediante la t de Student. Además, para comparar la distribución entre las tres zonas de Olson (equilibrado, valor medio o extremo) aplicamos el test de aleatorizacion de Cochrane. Los límites de significación son: tendencia $(\mathrm{p}<0,1)$, significativo $(\mathrm{p}<0,05)$ y altamente significativo $(\mathrm{p}<0,01)$. Todas las pruebas fueron a dos colas de la distribución.

Los resultados se presentan en el siguiente orden: análisis de variables demográficas (edad y género), discusión del impacto de esos parámetros, comparación de las puntuaciones en las dos poblaciones, validación del cuestionario de Olson, distribución de las dos poblaciones en las tres zonas y los dieciseis cuadrantes y sus correlaciones con el género y la edad. Otros parámetros demográficos clásicos tales como origen étnico, ocupación y nivel educacional no son relevantes para este estudio. El origen étnico y la ocupación se establecieron según los criterios de inclusión, y el nivel educativo está directamente relacionado con la edad en el diseño de este estudio.

\section{Resultados}

\section{Características demográficas}

\section{Distribución por edad}

El grupo "sano" estaba constituído por 814 sujetos entre 13 y 25 años, con una media de 18 años y una desviación estándar de 3 años. El grupo "enfermo" incluía a 358 sujetos con una edad media de 20,5 años (rango de 14 a 25 años ) y una desviación estándar de 3 años. Las diferencias fueron estadísticamente significativas $(\mathrm{t}=-13,179$, $\mathrm{p}<0,000)$.

\section{Distribución por género}

La proporción era de 329 hombres y 436 mujeres $(0,75)$ en el grupo "sano", y de 129 hombres y 229 mujeres $(0,56)$ en el grupo "enfermo". Estas diferencias son estadísticamente significativas $\left(\chi^{2}\right.$ de Pearson $=$ 69,112, $\mathrm{p}<0,000)$.

\section{Impacto de variables demográficas}

Con independencia de las hipótesis, que implican un estudio de correlación con la edad y el género, observamos que las diferencias en las distribuciones entre los dos grupos eran lo suficientemente importantes para incorporan dichas variables en los análisis.

\section{Verificación de hipótesis}

Diferencias en cohesión y adaptabilidad entre los dos grupos

La Tabla III muestra los resultados de la prueba t de Student. Se documenta claramente que existen diferencias significativas entre las familias del grupo "sano" y las del grupo "enfermo". Los adolescentes "enfermos" tienen familias menos adaptables y cohesionadas.

\section{Validación de las normas de Olson}

Para estudiar la distribución de nuestras dos poblaciones en términos de la tipología de Olson, y dada la manera en la que Olson (1986) estableció estas normas (basadas en una población adulta de norteamericanos), pensamos que era importante recalcularlas para nuestro rango de edad y nuestra población. Además de esto, para mantener la validez externa de la prueba, también calculamos las normas para todos nuestros sujetos combinados, por dos razones: 
- Aunque podemos excluir la posibilidad de que un sujeto "no enfermo" esté oculto dentro de la población "enferma", no podemos estar seguros de que la población sana es "pura". De hecho, es posible que un sujeto "enfermo" esté oculto en nuestra población "sana", dado que no se llevó a cabo una entrevista psiquiátrica de cribado.

- Si solamente calculásemos las normas basándonos en los sujetos "sanos" estaríamos favoreciendo a un grupo desde el principio, considerándolo como normativo y, por consiguiente, estaríamos considerando nuestras hipótesis como verdaderas antes de comenzar.

Estas normas son, consiguientemente: cohesión: 32 (d.e. $=8, \mathrm{n}=1.101)$, y adaptabilidad: 26 (d.e. $=6, \mathrm{n}=1.101$ ).

Distribución de los dos grupos en tres zonas y dieciseis cuadrantes

Para ver si podíamos ir más allá en la clasificación de nuestros dos grupos, intentamos comprobar si la distribución en las tres zonas

Tabla III

Prueba t para las familias "sanas"/“enfermas" de Olson

\begin{tabular}{lcccccc} 
Olson & $\mathrm{t}$ & $\mathrm{g} .1$. & $\mathrm{p}$ & media & d.e. & $\mathrm{N}$ \\
\hline $\begin{array}{l}\text { Adaptabilidad } \\
\text { "sanos" }\end{array}$ & 3.432 & 1.055 & 0,001 & & & \\
$\begin{array}{l}\text { "enfermos" } \\
\text { Cohesión }\end{array}$ & & & & 26,5 & 5,7 & 716 \\
"sanos" & 11,6 & 1.099 & 0,000 & 25,2 & 6,3 & 341 \\
"enfermos" & & & & 33,8 & 7,1 & 756 \\
\hline
\end{tabular}

de Olson era diferente, al mismo tiempo que su orden en la clasificación. La prueba de Cochrane confirmó esta hipótesis (Tabla IV).

Finalmente, si estudiamos las diferencias en la distribución entre los grupos para cada uno de los dieciséis cuadrantes de Olson, representando cada una de las tres posibles distribuciones en el modelo de Olson (significativo con la mayoría de "enfermos"; no significativo; significativo con la mayoría de "sanos") por un diferente color de fondo, obtenemos una figura (Figura 1) en la que está claro que los cuadrantes estructurado y rígido-desligado contienen más pacientes "enfermos". Estos resultados coinciden parcialmente con los de Lowler (1990), Prange (1992) y Tubiana (1998), refiriéndonos a un impacto significativamente alto de la cohesión en la adaptabilidad.

\section{Influencia del género y la edad}

Uno de los objetivos de este estudio era comprobar si el género y la edad jugaban un papel o no. Independientemente de si

Tabla IV

Prueba de Cochrane aplicada a las zonas de Olson para "sanos" / "enfermos"

\begin{tabular}{lcccc} 
Zonas de Olson & N "sanos" & N "enfermos" & $\%$ "sanos” & $\%$ "enfermos" \\
\hline Categoría equilibrada & 329 & 133 & $46 \%$ & $39 \%$ \\
Categoría media & 289 & 140 & $41 \%$ & $41 \%$ \\
Categoría extrema & 92 & 68 & $13 \%$ & $19 \%$ \\
\hline
\end{tabular}

$\mathrm{p}=0,002$ 
estudiábamos o no el efecto del género de la población total de individuos de los grupos "sano" o "enfermo", no hallamos ninguna influencia. Por otra parte, se encontró que la edad era un factor determinante, ya que observamos una disminución de la cohesión familiar con la edad (Tabla V), excepto en el grupo "enfermo".

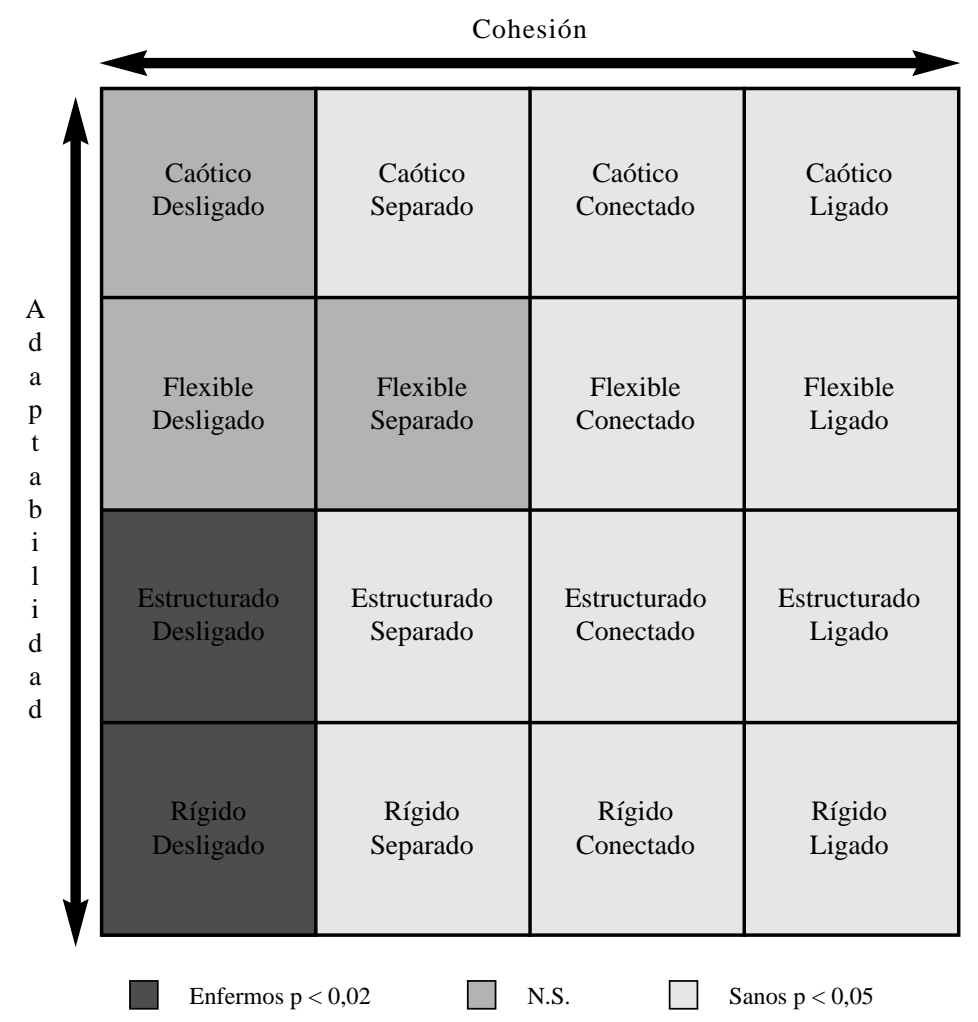

Figura 1. Puntos de corte significativo entre cuadrantes en "enfermos" / "sanos", según el modelo de Olson.

\section{Discusión}

Como se ha demostrado en este estudio, hay claras diferencias entre los dos grupos, el "sano"y el "enfermo". Estos resultados confirman los estudios previos y sugieren dos teorías adicionales. En primer lugar, independientemente de la "enfermedad" que el adolescente tenga, recae estadísticamente más a menudo en algunas de las áreas patológicas descritas por Olson (que son “estructurado"-“desligado" y "rígido"-“desligado") y, en segundo lugar, hay un aspecto evolutivo por el que, al menos en el grupo "sano", observamos una menor cohesión en función de la edad.

La observada "marginalización" (en términos de los modelos de Olson) para los adolescentes "enfermos" puede interpretarse de dos maneras. O, desde un punto de vista general, tener una familia con poca cohesión y adaptabilidad representa un ries- 
Tabla V

Variables de Olson en función de la edad

\begin{tabular}{lccc} 
Muestra total & $\mathrm{r}$ & $\mathrm{p}$ & $\mathrm{N}$ \\
\hline Adaptabilidad familiar & $-0,066$ & 0,035 & 1.037 \\
$\begin{array}{l}\text { Cohesión familiar } \\
\text { Grupo "sano" }\end{array}$ & $-0,179$ & 0,000 & 1.079 \\
Adaptabilidad familiar & $-0,018$ & 0,542 & 696 \\
Cohesión familiar & $-0,097$ & 0,008 & 734 \\
Grupo "enfermo" & $-0,056$ & 0,299 & 341 \\
Adaptabilidad familiar & $-0,011$ & 0,841 & 345 \\
Cohesión familiar & &
\end{tabular}

go adicional de padecer cualquier trastorno psicopatológico, o bien el hecho de que el adolescente esté padeciendo una "enfermedad", no importa cuál, cambia las interacciones familiares. Claramente, aquí no es posible distinguir entre estas dos explicaciones ya que, que nosotros sepamos, no se ha realizado ningún estudio prospectivo utilizando el modelo de Olson. Es muy posible que los dos tipos de interacciones existan y, desde un punto de vista evolutivo, esto apoyaría las teorías familiares, como previamente sugirieron Lundholm et al. (1991).

La disminución de la cohesión familiar en función de la edad en el grupo "sano" podría interpretarse de tres maneras diferentes:

1. Con el paso del tiempo, el sistema familiar es menos cohesivo, $\mathrm{o}$

2. Con el paso del tiempo, el adolescente llega a ser capaz de diferenciarse a sí mismo del sistema, por eso se introduce cierta "incoherencia", o

3. Debería recordarse que nuestros instrumentos evalúan el sistema familiar a través de la percepción de los adolescentes sobre sus familias y, por consiguiente, la interpretación de la percepción del adolescente puede cambiar, con el tiempo, hacia cierta "desidealización" del modelo familiar. El adolescente llega a ser consciente de que su familia es menos coherente de lo que a él le hubiera gustado creer cuando era un niño.

Puesto que, afortunadamente, no todas las familias se divorcian, tendemos a defender la segunda y tercera interpretaciones. Éstas interpretaciones entran en el terreno de la investigación psicodinámica, que proclama que la adolescencia es la etapa final de la creación del individuo como un sistema psíquico independiente (Cahn 1998). Nos gustaría también enfatizar que esta disminución no se observa en el grupo de adolescentes "enfermos", lo que sugiere que, para crecer y separarse de la familia, se requiere una familia cohesiva.

\section{Limitaciones y conclusión}

La principal limitación de la validez de nuestros resultados es la duración del reclutamiento de la muestra de "enfermos". Ciertamente, el hecho de tener resultados controlados por fecha de hospitalización no nos permite excluir totalmente modificaciones socioculturales, farmacológicas u otras que pudieran haber influenciado nuestros resultados.

Atendiendo a estas observaciones, creemos que este estudio puede tener al menos 
dos implicaciones prácticas (que deben, por supuesto, ser estudiadas mediante un diseño prospectivo):

En primer lugar, pensamos que el cuestionario de Olson es un instrumento que permitirá una valoración del pronóstico. De esta manera, un adolescente "enfermo" que puede ser situado dentro del cuadrante "normal" del modelo de Olson tendrá, sin lugar a dudas, un mejor pronóstico que un adolescente cuya familia se sitúa en los cuadrantes extremos o, incluso peor, en las áreas "desligado" - "rígido" o "desligado"- "estructurado". Si esta hipótesis se confirma, podríamos tener incluso un instrumento preventivo a nuestra disposición. Por ejemplo, un adolescente que adopta un patrón de conductas de riesgo (fumar, abuso de alcohol...) sería más o menos propenso a perseverar en dicha conducta, dependiendo de las interacciones con su familia.

En términos de tratamiento, este estudio también sugiere que, al tratar adolescentes con trastornos, también es necesario investigar las interacciones familiares con el fin de hacerlas más coherentes y adaptables. Finalmente, persiste el hecho de que es necesario un salto conceptual entre las interacciones familiares por un lado, y la salud por el otro. Por consiguiente, proponemos investigar en el futuro las relaciones entre estos dos factores utilizando el MLHC y esperamos que así se aclarará uno de los eslabones que faltan, sin duda numerosos.

\section{Bibliografía}

BEAVERS, W.R. Healthy, midrange and severely dysfunctional families. In F. Walsch (Ed): Normal Family Process. Guilford, New York, 1982.

CAHN, R. L'adolescent dans la psychanalyse. PUF, Paris, 1998.
COBURN, J., GANONG, L. Bulimic and non-bulimic college females' perceptions of family adaptability and family cohesion. Journal of Advanced in Nursering 14, 27 33, 1989.

DSM IV. Diagnostic and Statistical manual of mental disorders, fourth edition. Washington: American Psychiatric Association Press, 1994.

FONTAINE, P. Famille saines; esquisse conceptuelle générale. Thérapie Familiale 3, 267-282, 1985.

JANNE, P. De la douleur à la plainte: pour une compréhension psychosomatique de l'ischémie myocardique dite silencieuse: thèse de doctorat. Faculté de Psychologie et des Sciences de l'Education, Université Catholique de Louvain, 1989.

KASHANI, J.H., ALLAN, W.D., DAHLMEIER, J.M., REZVANI, M., REID, J.C. An examination of family functioning utilizing the circumplex model in psychiatrically hospitalizad children with depression. Journal of Affective Disorders 35, 65-73, 1995.

KING, S., DIXON, M.J. Expressed emotion, family dynamics and symptom severity in a predictive model of social adjustment for schizophrenic young adults. Schizophrenia Research 14, 121-132, 1995.

LAWLER, M.K., VOLK, R., VIVIANI, N., MENGEL, M.B. Individual and family factors impacting diabetic control in the adolescent: a preliminary study. Maternity and Child Nursing Journal 19, 331-345, 1990.

LUNDHOLM, J.K., WATERS, J.E. Dysfunctional family systems: relationships to disordered eating behaviors among university women. Journal of Substance Abuse 3, 97-106, 1991.

MICHAELS, C.R., LEWANDOWSKI, L.J. Psychological adjustment and family functioning of boys with learning disabilities. Journal of Learning Disability 23, 446450, 1990.

OLSON, D.H. Circumplex model: validation studies FACES III. Family Process 25, 337-351, 1986.

OLSON, D.H., KILLORIN, E. Clinical rating scale for circumplex model (revised version). StPaul MN: Family Social Science, University of Minnesota, 1985.

O.M.S. (WHO). La santé des jeunes. OMS, Genève, 1994.

PRANGE, M.E., GREENBAUM, P.E., SILVER, S.E., FRIEDMAN, R.M., KUTASH, K., DUCHNOWSKI, A.J. Family functioning and psychopathology among adolescents with severe emotional disturbances. Journal of Abnormal Child Psychology 20, 83-102, 1992. 
REYNAERT, CH., JANNE, P., FONTAINE, P., WOERLINCK, K., COLLIN, A., CASSIERS, L. Eléments pour une reconsidération circulaire du modèle circomplexe de Olson ou: comment la logique des contraire est aussi une logique des proximités. Thérapie Familiale 12, 55-63, 1991.

REYNAERT, CH., JANNE, P., ZDANOWICZ, N., VAUSE, M., VERVIER, JF. Description du système familial par ses membres: le sentiment d'autonomie. Cahier des Sciences Familiale et Sexologique 17, 95-111, 1993.

TUBIANA, R.N., MORET, L., CZERNICHOW, P. CHWALOW, J. The association of peor adherence and acute metabolic disorders with low levels of cohesion and adaptability in families with diabetic children. Acta Paediatrica 87, 741-746, 1998.

WALLSTON, K.A., WALLSTON, B.S., DE VELLIS, R. Development of the multidimensional health locus of control (MHLC) scale. Health Education Monography 6, 160-170, 1978.

YOUNGBLUT, J.M., SHASO, S.Y. Child and family reactions during and after pediatric ICU hospitalization: a pilot study. Hearth \& Lung: The Journal of Critical Care 22, 46-54, 1993.

ZDANOWICZ, N., JANNE, P., REYNAERT, CH. Lieu de controle de la santé et prise en charge des adolescents. Revue Française de Psychiatrie et de Psychologie Médicale 54, 20-25, 2002.

ZDANOWICZ, N., JANNE, P., REYNAERT, CH. Changes in the health locus of control during adolescence of student. The European Journal of Psychiatry 2, 107115, 2003.

Dirección para correspondencia:

N. Zdanowicz, MD

Université Catholique de Louvain

Clinique de Mont-Godinne

Service de psychosomatique

5530 Yvoir

BELGIQUE - BELGIUM

E-mail: nicolas.zdanowicz@pscl.ucl.ac.be 